\title{
Novel Object Recognition as a Facile Behavior Test for Evaluating Drug Effects in A $\beta P P / P S 1$ Alzheimer's Disease Mouse Model
}

\author{
Ru Zhang ${ }^{\mathrm{a}, 1}$, Guizhen Xue ${ }^{\mathrm{a}, 1}$, Shaodeng Wang ${ }^{\mathrm{a}}$, Lihong Zhang ${ }^{\mathrm{a}}$, Changjie Shi ${ }^{\mathrm{a}}$, Xin Xie $\mathrm{Xi}^{\mathrm{a}, \mathrm{b}, *}$ \\ ${ }^{a}$ Shanghai Key Laboratory of Signaling and Disease Research, Laboratory of Receptor-based Bio-medicine, \\ School of Life Sciences and Technology, Tongji University, Shanghai, China \\ ${ }^{\mathrm{b}}$ State Key Laboratory of Drug Research, The National Center for Drug Screening, Shanghai Institute \\ of Materia Medica, Chinese Academy of Sciences, Shanghai, China
}

Handling Associate Editor: Zhi-Ying Wu

Accepted 14 May 2012

\begin{abstract}
Alzheimer's disease (AD) is a progressive neurodegenerative disorder and the A $\beta P P / P S 1$ transgenic mouse model is a commonly used experimental model to mimic the pathological and cognitive impairments in AD. As a classic method to evaluate spatial learning and memory, the Morris water maze is widely applied to study the cognitive deficits in rodent AD models. However, the assay procedure is relatively complicated and requires a properly equipped environment. The novel object recognition test is a relatively simple and straightforward method to test working memory in rodents. However, whether the latter can be used as a common tool for evaluating the therapeutic effects of drugs in the AßPP/PS1 transgenic AD mouse model remains unclear. In the present study, we assessed the cognitive impairment of A $\beta P P / P S 1$ AD mice with the novel object recognition test. In parallel, Morris water maze was performed and compared with the novel object recognition study. Both assays worked equally well in evaluating the cognitive defect of AßPP/PS1 mice. Furthermore, we drew similar conclusions from the novel object recognition assay as from the Morris water maze in assessing the therapeutic effects of two previously reported compounds, donepezil and naltrindole, on $\mathrm{AD}$. We found the novel object recognition to be a facile assay with almost no stress to mice and think it could be used as an ideal primary screening assay to evaluate drug effects on AßPP/PS1 AD model.
\end{abstract}

Keywords: A $\beta P P / P S 1$, Alzheimer's disease, donepezil, Morris water maze, naltrindole, novel object recognition

\section{INTRODUCTION}

Alzheimer's disease (AD) is a progressive neurodegenerative disorder, with cognitive, memory, and behavioral impairments. In $\mathrm{AD}$ patients, plaques

\footnotetext{
${ }^{1}$ These authors contributed equally to this study.

*Correspondence to: Xin Xie, Ph.D., Shanghai Institute of Materia Medica, 189 Guo Shou Jing Rd, Shanghai 201203, China. Tel.: +86 2150801313 ext. 156; Fax: +86 2150800721 ; E-mail: xxie@mail.shcnc.ac.cn.
}

composed of the aggregated amyloid $\beta$-peptide $(\mathrm{A} \beta)$ and tangles containing the hyperphosphorylated tau protein distribute across certain regions of the brain [1]. In order to mimic the pathological development of $\mathrm{AD}$, several mouse models have been introduced. Similar to AD patients, these mice suffer from progressive decline in learning and memory. One popular AD model is the amyloid- $\beta$ protein precursor/presenilin 1 $(\mathrm{A} \beta P P / P S 1)$ double transgenic mice. These mice carry a mouse/human chimeric A $\beta P P$ containing the 
K595 N/M596 L Swedish mutations and the exon 9deleted form of human PS1, a core component of the $\gamma$-secretase complex. Increased sequential cleavage of A $\beta P P$ by $\beta$-secretase and $\gamma$-secretase promotes $A \beta$ generation and plaque formation. An age-dependent increase of $\mathrm{A} \beta$ depositions has been documented in A $\beta P P / P S 1$ mice and these animals display cognitive deficits at the age of 6 months [2,3].

Many behavioral tests have been created to evaluate the cognitive deficits relevant to $\mathrm{AD}$ pathology in rodent models. The Morris water maze is the most commonly applied method to test the spatial memory in rodents [4]. Tests concerning contextual memory, such as fear conditioning, is widely used to measure hippocampal functions [5] and can also be applied in AD mouse models [6]. Y-maze and T-maze, in combination with positive or negative stimulus which help to define conditional, trial-specific information that is used by the animals to select between response options [7], are often used to test working memory in AD animal models [3, 8]. The novel object recognition test was first proposed by Ennaceur and Delacour in 1988. This test is based on the spontaneous behavior of rats to interact more with a novel object than with a familiar one [9]. Unlike Morris water maze, this test does not concern the spatial reference memory of the animals. The experiment consists of two phases. In the training phase, rats are allowed to explore two identical objects. In the testing phase, one of the two identical objects is replaced by a novel one. Depending on the length of the delay between the two trials, the rats will either explore the novel object for a longer time period, indicating memory for the familiar object; or will explore the novel and familiar objects for the same amount of time, indicating a lack of recall or loss of memory for the familiar object presented during the initial trial. This assay was used to detect the deficits in learning and memory in scopolamine- or $\mathrm{A} \beta$ peptide-induced $\mathrm{AD}$ models $[10,11]$, or transgenic (TgCRND8 and Tg2576) AD models [12, 13]. A recent report also used novel object recognition to show cognitive improvement by compound treatment in the A $\beta P P / P S 1$ mice [14]. However, no detailed comparison has been made between the classic Morris water maze and novel object recognition tests, and whether or not the latter can be used as a common tool for evaluating the therapeutic effects of drugs in $\mathrm{A} \beta \mathrm{PP} / \mathrm{PS} 1$ transgenic $\mathrm{AD}$ mouse model remains unclear.

In the present study, we assessed the cognitive impairment of A $\beta P P / P S 1$ AD mice with the novel object recognition test. In parallel, Morris water maze was performed and compared with the novel object recognition study. Both assays worked equally well in evaluating the cognitive deficits in A $\beta P P / P S 1$ mice. Furthermore, the novel object recognition assay led to similar conclusions as the Morris water maze in assessing the therapeutic effects of two previously reported compounds, donepezil and naltrindole (NTI), on AD. We found novel object recognition to be a facile assay with almost no stress to mice, and that it could be used as an ideal primary screening assay to evaluate the drug effects on A $\beta P P / P S 1$ AD model.

\section{MATERIALS AND METHODS}

\section{Animals}

A $\beta P P / P S 1$ mice carrying a chimeric mouse/human AßPP with the K595 N/M596 L Swedish mutations and the exon-9-deleted variant of human PS1 on a C57BL/6 background were obtained from Jackson Laboratory (stock number 004462). Wild type (WT) littermates were used as control. Animals were housed up to 5 in each cage under the standard conditions. Mice had free access to food and water under a $12 \mathrm{~h} / 12 \mathrm{~h}$ light/dark cycle (light on from 09: 00 to 21 : 00). Oneweek before behavioral tests, animals were habituated in a room with reversed $12 \mathrm{~h} / 12 \mathrm{~h}$ light/dark cycle (light off from 09:00 to $21: 00$ ). All procedures were in accordance with institutional guidelines for animal care and administration.

\section{Drug treatment}

Donepezil hydrochloride and NTI were obtained from Eisai China Inc., and Sigma, respectively. The drugs were dissolved in distilled water. Four month old AD mice were randomly divided into 3 groups. Vehicle group ( $n=10,6$ males and 4 females) received distilled water daily $(0.2 \mathrm{ml}$ per mouse). The remaining 2 groups received daily oral administration of donepezil ( $2 \mathrm{mg} / \mathrm{kg}, n=11,6$ males and 5 females $)$ or NTI $(5 \mathrm{mg} / \mathrm{kg}, n=13,7$ males and 6 females) for 3 months. Their WT littermates $(n=14,7$ males and 7 females) received water orally. Behavioral tests were performed after 3 months of treatment. The treatment continued during the tests until the mice were sacrificed.

\section{Novel object recognition}

The novel object recognition test was modified from a previously described method [13]. The 


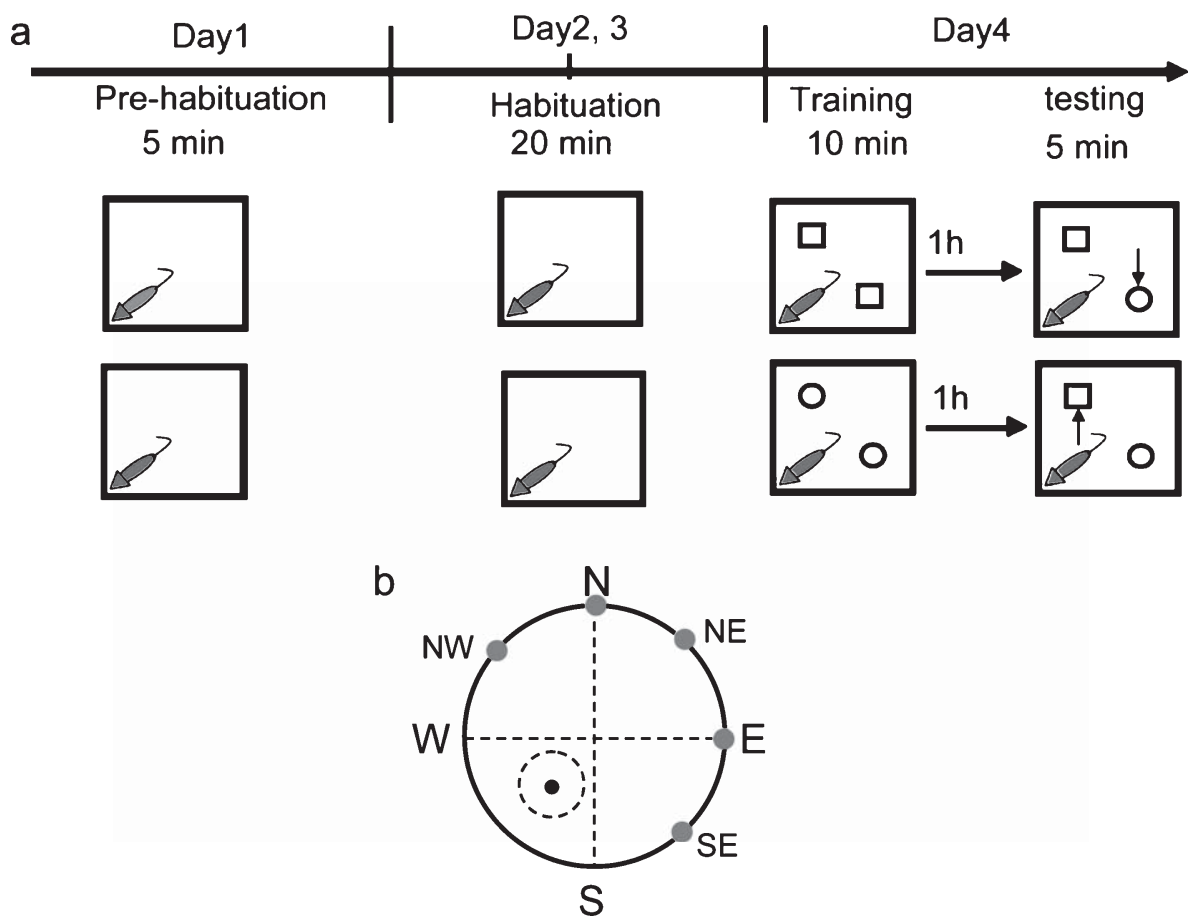

Fig. 1. a) Schematic representation of novel object recognition assay. Following pre-habituation and habituation in the empty boxes, mice were allowed to explore an identical pair of objects placed in the boxes for $10 \mathrm{~min}$ as the training session. After $1 \mathrm{~h}$ stay in the home cage, the mice were returned to the arena where two objects, one familiar and one novel, were placed. The time mice spent in exploring the two objects was recorded. The arrows indicate the novel objects in the two boxes. b) The diagram of the Morris water maze pool with the platform (black dot) and the five starting positions in daily trials and the probe trial (gray dots). The dash lines separate the four quadrants of the pool and the dash line circle indicates the area of five times the platform diameter centered at the platform location.

detailed procedures are schematically represented in Fig. 1a. The apparatus consists of an evenly illuminated sound-proof box with a plexiglass box $(25 \mathrm{~cm} \times 25 \mathrm{~cm} \times 25 \mathrm{~cm})$ inside. The mouse behavior is recorded with a video camera. The procedure includes 4 phases: pre-habituation, habituation, training, and testing. On the 1 st day, animals were brought to the testing room $30 \mathrm{~min}$ before the experiment to familiarize with the environment. Mice were then allowed to freely explore the box in the absence of objects for $5 \mathrm{~min}$. On the 2 nd and $3 \mathrm{rd}$ day, mice were habituated to the empty box for 20 min per day. On the 4th day, each mouse took a training trial followed by a testing trial. During the training trial, two identical objects were placed at two opposite positions within the box at same distance from the nearest corner. The mice were allowed to explore the identical objects for $10 \mathrm{~min}$ and then returned to their home cages. One hour later, animals were placed back to the same box, where one of the two familiar objects was switched to a novel one, to start a 5 min testing phase. All objects used in this study were different in shapes and colors but identical in size.
They were fixed on the floor of the box to avoid movement. To preclude the existence of olfactory cues, the entire box and objects were always thoroughly cleaned with water after each trial.

Object exploration time was defined as the length of time when animal directing its nose within $2 \mathrm{~cm}$ distance to the object, or sniffing or pawing the object. Sitting or standing on the object was not recognized as exploration. The exploration time was analyzed manually using 2 stop watches. In the training session, the location preference in the training phase and recognition index (RI) in the testing phase were calculated using the following formula:

Location preference $=$ Time exploring one of the identical objects/Time exploring the identical object pairs $\times 100 \%$

Recognition index $(\mathrm{RI})=$ Time exploring novel object/(Time exploring novel object + Time exploring familiar object) $\times 100 \%$

Location preference was used as an environmental control, which should be $50 \%$, to rule out the influence of the location of the object. Animals with a total 
exploration time of less than $3 \mathrm{~s}$ during testing session were excluded from analysis.

\section{Morris water maze}

The water maze consists of a circular pool $(1.2 \mathrm{~m}$ in diameter) filled with water $\left(22 \pm 1^{\circ} \mathrm{C}\right)$. White, nontoxic plastic beads were spread over the surface in order to make the water surface opaque and to have a good contrast to the black color of the C57BL/6 mice. A CCD camera was installed above the pool and connected to a computerized tracking system. Distal visual cues were fixed to the room walls. Four positions around the pool were artificially specified as north $(N)$, south (S), east (E), and west (W) and the pool was divided into four quadrants (Fig. 1b). In the hidden platform training session, a white platform $(8 \mathrm{~cm}$ in diameter) was placed in the middle of the SW quadrant and $1 \mathrm{~cm}$ beneath the surface of the water. Mice were trained 4 times a day. Each mouse was placed into the pool facing the wall with the start location at $\mathrm{N}, \mathrm{E}, \mathrm{NW}$, or SE positions, and the sequence of the starting location was randomly balanced. In each trial, the mouse was given a maximum of $60 \mathrm{~s}$ to escape to the platform. The animal succeeded in finding the platform within $60 \mathrm{~s}$ was kept on the platform for $15 \mathrm{~s}$. If the mouse failed to find the platform within $60 \mathrm{~s}$, it was guided to and allowed to stay on the platform for $15 \mathrm{~s}$. The training session was conducted for 6 consecutive days in which the platform was never moved. The latency to escape was calculated as the average time to find the platform of the 4 trials within one day. Memory retention was evaluated on the 7 th day with a probe trial in which the platform was removed. The mice were put into the pool at the NE position, and allowed to swim freely in the pool for $60 \mathrm{~s}$. The swimming paths of the mice were recorded. The time spent in the target quadrant where the platform used to be, or the area of five times the platform diameter centered at its former location was measured. Meanwhile, the times the mice crossed the area where the platform had been placed were counted and the swimming speed was calculated.

\section{Brain tissue preparation}

Mice were anesthetized with $10 \%$ chloral hydrate and perfused with saline. The brain was quickly removed and one hemisphere was dissected and fixed in freshly prepared $4 \%$ paraformaldehyde at $4{ }^{\circ} \mathrm{C}$ overnight. Then the brain hemisphere was soaked in $10 \%, 20 \%$, and $30 \%$ sucrose sequentially for
$24 \mathrm{~h}$. Tissue was embedded in tissue embedding medium (30\% sucrose, $30 \%$ ethylene glycol) and snap-frozen on dry ice. The brains were cut into $14 \mu \mathrm{m}$ thick serial sections in the coronal plane with a freezing microtome at $-20^{\circ} \mathrm{C}$. Tissue sections were then stored in the section protection solution at $-20^{\circ} \mathrm{C}$.

\section{Thioflavin $S$ staining for $A \beta$ aggregation}

Thioflavin S, a dye commonly used to stain $\beta$ sheet structures $[15,16]$, was used to visualize the amyloid plaque in cortex and hippocampus. A series of tissue sections of similar brain regions from different mice were selected for staining. The sections were washed 3 times with $0.1 \mathrm{M}$ phosphate buffer (PB, $0.1 \mathrm{M} \mathrm{KH}_{2} \mathrm{PO}_{4}$ and $0.08 \mathrm{M} \mathrm{NaOH}, \mathrm{pH}=7.4$ ) for $5 \mathrm{~min}$ and then incubated in $0.1 \%$ thioflavin $\mathrm{S}$ for $8 \mathrm{~min}$ followed by washing in $70 \%$ ethanol for $5 \mathrm{~min}$. Then the tissue sections were incubated in PB for $5 \mathrm{~min}$ and mounted on the slides. Images were acquired using Olympus IX71 fluorescence microscope. The thioflavin $\mathrm{S}$ positive area in the brain sections was analyzed using Image-Pro Plus 5.1 software. All mice were subjected to the staining and for each mouse, $6 \sim 8$ sections of each brain region were analyzed.

\section{Immunohistochemistry}

After washing thrice with $\mathrm{PB}$, the brain sections were permeabilized in antibody dilution buffer $(0.2 \%$ Triton X-100 and 1\% BSA in PB) for $20 \mathrm{~min}$ at room temperature. Then the brain sections were incubated with rat anti-GFAP primary antibody $(1: 2000$, Invitrogen) overnight at $4{ }^{\circ} \mathrm{C}$. After washing, the sections were incubated with Alexa Fluor 488-congjugated goat anti-rat antibody $(1: 1000$, Invitrogen) for $1 \mathrm{~h}$ at room temperature in the dark. The percentage of GFAP positive area in the sections was analyzed using Olympus IX71 fluorescence microscope and Image-Pro Plus 5.1 software. All mice were subjected to the immunohistochemistry staining and for each mouse, $6 \sim 8$ sections of each brain region were analyzed.

\section{Statistical analysis}

All data were expressed as means \pm S.E.M. Twoway ANOVA test was used to analyze the escape latency in Morris water maze. The statistical significance of other comparisons was analyzed with 
one-tailed Student's $t$-test. $p<0.05$ was considered as statistically significant.

To further establish the correlation between the novel object recognition and Morris water maze experiments, we introduced scatter plot to analyze the data of each mouse obtained from the two behavioral tests. Xaxis represents the recognition index obtained from the novel object recognition and Y-axis represents various performance parameters of the same mouse in Morris water maze, including platform location crosses, time in target quadrant, and time in the area of five times the platform diameter centered at the platform location. Mice with data points located in the lower left field showed memory deficits in both tests, while mice with data points located in the upper right field performed good in both tests.

a
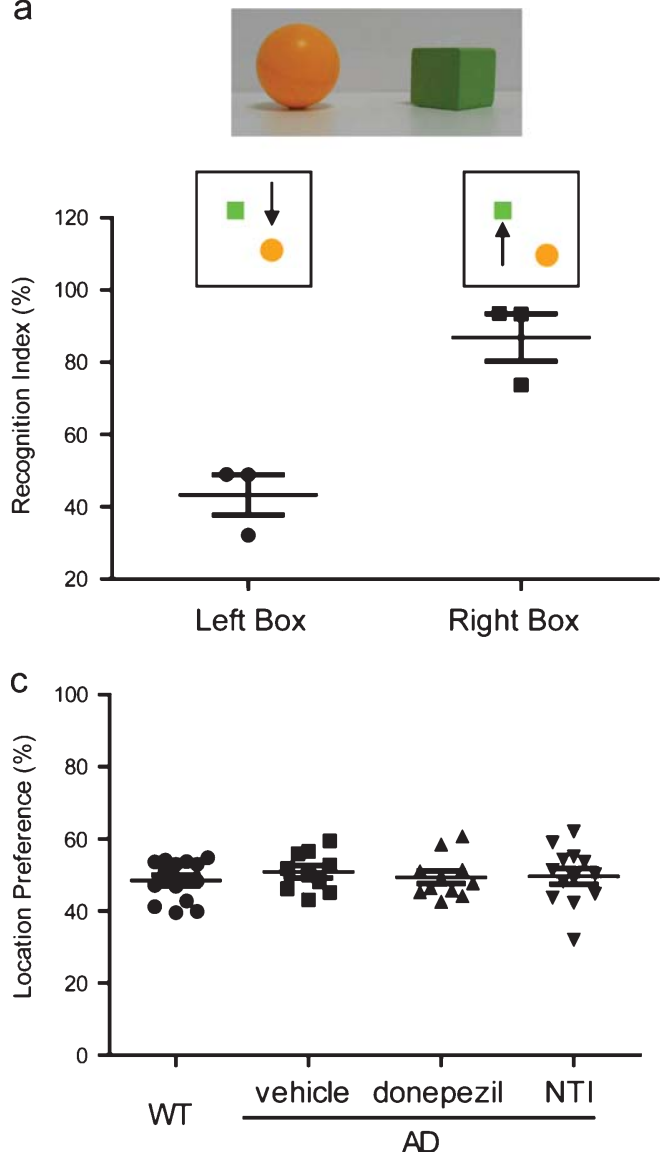

\section{RESULTS}

Selection of suitable object pairs for the novel object recognition test

Different features of the objects, such as size, shape, material, presence of protrusions/intrusions, texture of the surface and brightness of the color, greatly influence the interaction between the rodents and the objects [17-20]. To make sure that the mice were not attracted more to one object than the other, we carefully selected the object pairs with the protocol shown in Fig. 1a with WT mice. We chose objects with a simple shape, washable materials (e.g., odorless plastic or metal), similar size as mice, and colors with comparable brightness for pre-screening. As shown in Fig. 2a,

b
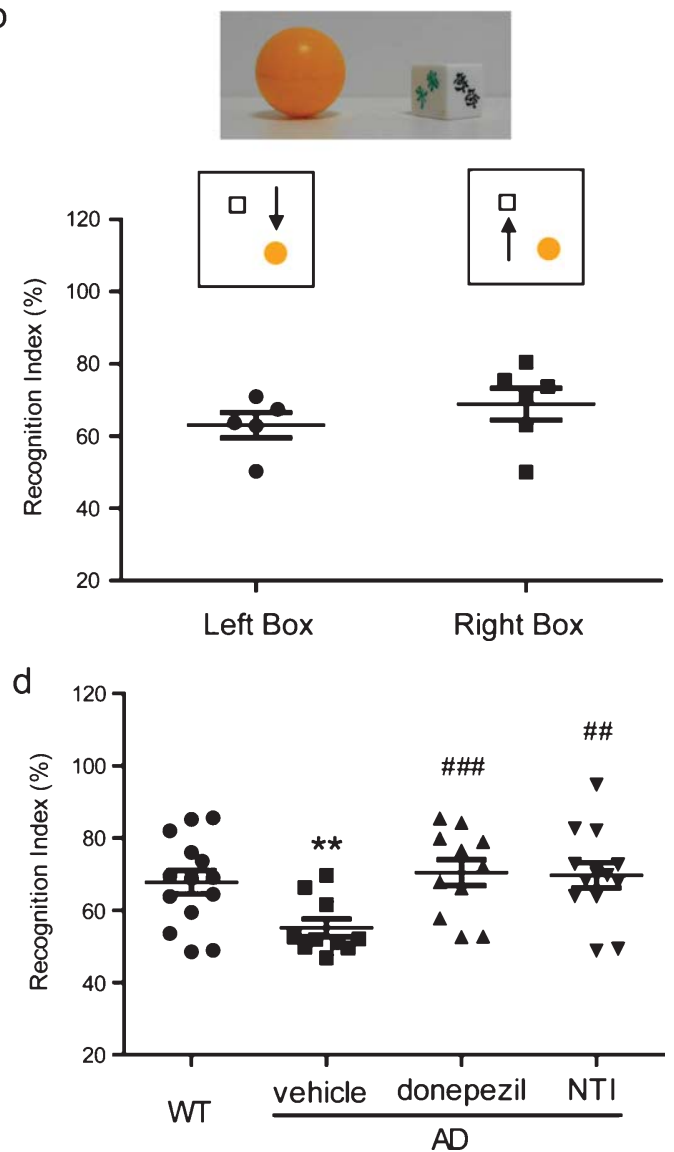

Fig. 2. Donepezil and NTI treatment improve the recognition index of AßPP/PS1 mice in novel object recognition test. Various object pairs were pre-screened according to the protocol described in Fig. 1. a) Example of unsuitable object pairs. Mice preferred one object (the green wooden cube) in the pair. b) Example of suitable object pairs. Mice showed no preference of the object, but explored the novel one (arrow) more. c, d) Performance of mice in novel object recognition after donepezil and NTI treatment. WT littermates $(n=14)$ and AD mice treated with vehicle $(n=10)$, donepezil $(n=11)$, or NTI $(n=13)$ were subjected to the novel object recognition test using pre-selected object pairs. c) The location preference is calculated as the percentage of time a mouse spent in exploring one of the two identical objects within the 10 min training session. d) The recognition index is calculated as the percentage of time a mouse spent exploring the novel object in the 5 min testing session. Data represent mean \pm S.E.M. ${ }^{*} p<0.01$ versus WT; ${ }^{\# \#} p<0.01$ and ${ }^{\# \# \# ~} p<0.001$ versus AD vehicle controls (Student's $t$-test). 
the green wooden cube and plastic sphere were not a good pair since no matter which object was chosen as the novel one (arrow), the mice always preferred the wooden cube. In contrast, when using the plastic dice and plastic sphere as an object pair, the mice spent more time exploring the novel object (arrow) and gave similar recognition index regardless of the objects (Fig. 2b). Many object pairs were tested and only the suitable ones were used in the experiments below.

\section{Novel object recognition is a facile behavior test for evaluating drug effects in A $\beta P P / P S 1$ mouse model}

We used two chemicals, donepezil, a clinically approved drug (trade name Aricept) for AD, and NTI, a DOR antagonist reported to alleviate the behavioral deficits of AD mice in Morris water maze [21], to validate the performance of novel object recognition assay. The wild type littermates were given water as control. The assay was carried out according to the timeline shown in Fig. 1a. We found no significant difference in the time that mice spent exploring the two identical objects placed in diagonal locations during the training phase, indicating the location of the objects does not affect the exploration behavior of mice (Fig. 2c). In the testing phase with two different objects (one novel, the other familiar), WT mice explore the novel object for a longer time period with RI at approximately $68 \%$, indicating their memory for the familiar object. In contrast, AD mice treated with vehicle had a significantly lower RI at 55\%, which is consistent with their impaired cognitive function. When given daily for 3 months, both donepezil $(2 \mathrm{mg} / \mathrm{kg})$ and NTI $(5 \mathrm{mg} / \mathrm{kg})$ significantly increased the RI in AD mice to approximately $70 \%$ and $71 \%$, respectively, reflecting the therapeutic effect of these drugs on AD animals (Fig. 2d).

In parallel, we tested the mice in the classic Morris water maze to compare the performance of these two different methods. The average latencies for the mice to find the platform in the water maze are shown in Fig. 3a. All mice showed diminishing latency over the 6 training days with the first day at approximately $50 \mathrm{~s}$. As expected, WT mice showed significantly shorter escape latency than the AD mice treated with vehicle $(p<0.001)$, confirming the impaired spatial learning in the $\mathrm{AD}$ mice. Compared to the $\mathrm{AD}$ vehicle group, the NTI group showed significantly decreased escape latency, indicating the improvement in spatial learning $(p<0.01)$. Donepezil also significantly reduced the escape latency in $\mathrm{AD}$ mice, which is in agreement with its well-established therapeutic effect in $\mathrm{AD}$ patients. In the probe trial, the escape platform was removed and the swimming path of each mouse was recorded and analyzed. The WT mice showed a focal search in the target quadrant where the platform used to be located. Similar search patterns were observed in NTI- or donepezil-treated mice. In contrast, the vehicle-treated AD mice showed an aimless searching strategy (Fig. 3b). The analysis of platform crossing, time in target quadrant and time in the area of five times the platform diameter all showed a significant difference between WT and $\mathrm{AD}$ vehicle group (Fig. 3c-e). Donepezil-treated $\mathrm{AD}$ mice showed a significant increase in the number of platform crossing compared to the vehicle control group, whereas the NTI-treated group showed a similar tendency. However, both drug-treated groups showed only an increased tendency in time spent in the target quadrant or in the area of five times the platform diameter, suggesting the lack of sensitivity in this method. There was no significantly difference in swim speed between groups (Fig. 3f). These data suggests that the results from the novel object recognition test is consistent with the results generated from Morris water maze, and that the novel object recognition test is an easier and potentially more sensitive method to evaluate compound effect.

\section{NTI or donepezil treatment alleviates the pathological signs in the brain of A $\beta P P / P S 1$ mice}

$\mathrm{A} \beta$ deposition and astrocyte activation in the brain are typical pathological signs of AD patients and animal models. The A $\beta P P / P S 1$ mice have been reported to show $A \beta$ deposition in the cortex and hippocampus at 6 months of age [2]. The mice used in the behavioral tests were sacrificed and $A \beta$ deposition and astrocyte activation were investigated to confirm the therapeutic effects of the drugs. AD mice developed a significant numbers of plaques at 8 months, while no A $\beta$ plaque was observed in the WT mice (Fig. 4a). NTI-treated mice had significantly less $A \beta$ deposits in both cortex and hippocampus (Fig. 4a, c and d), which agreed with our results from behavioral tests and previously proposed mechanism that NTI reduces $A \beta$ generation [21]. Donepezil, as an acetylcholinesterase (AchE) inhibitor, showed less prominent influence on $A \beta$ plaque formation in hippocampus but significantly reduced the plaque formation in cortex. $A \beta$ 


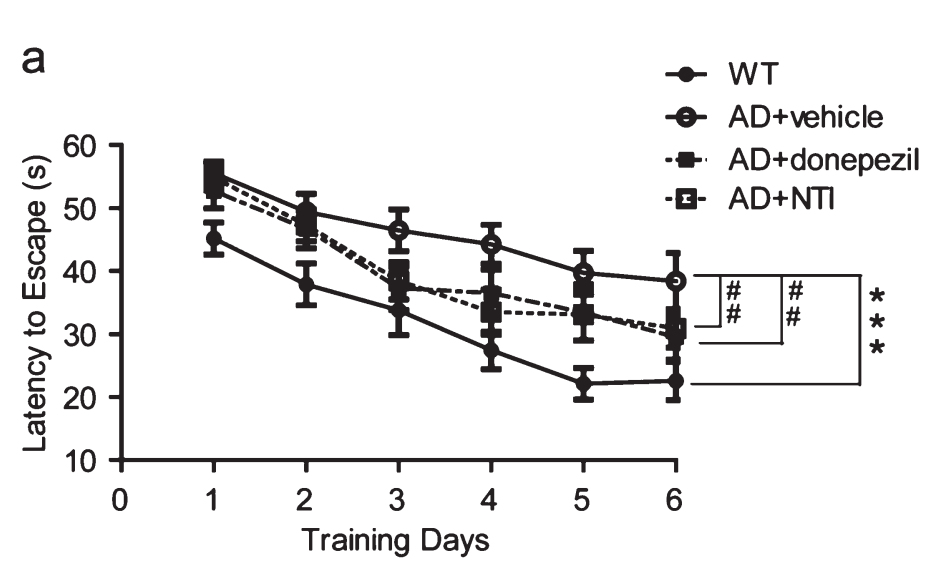

b
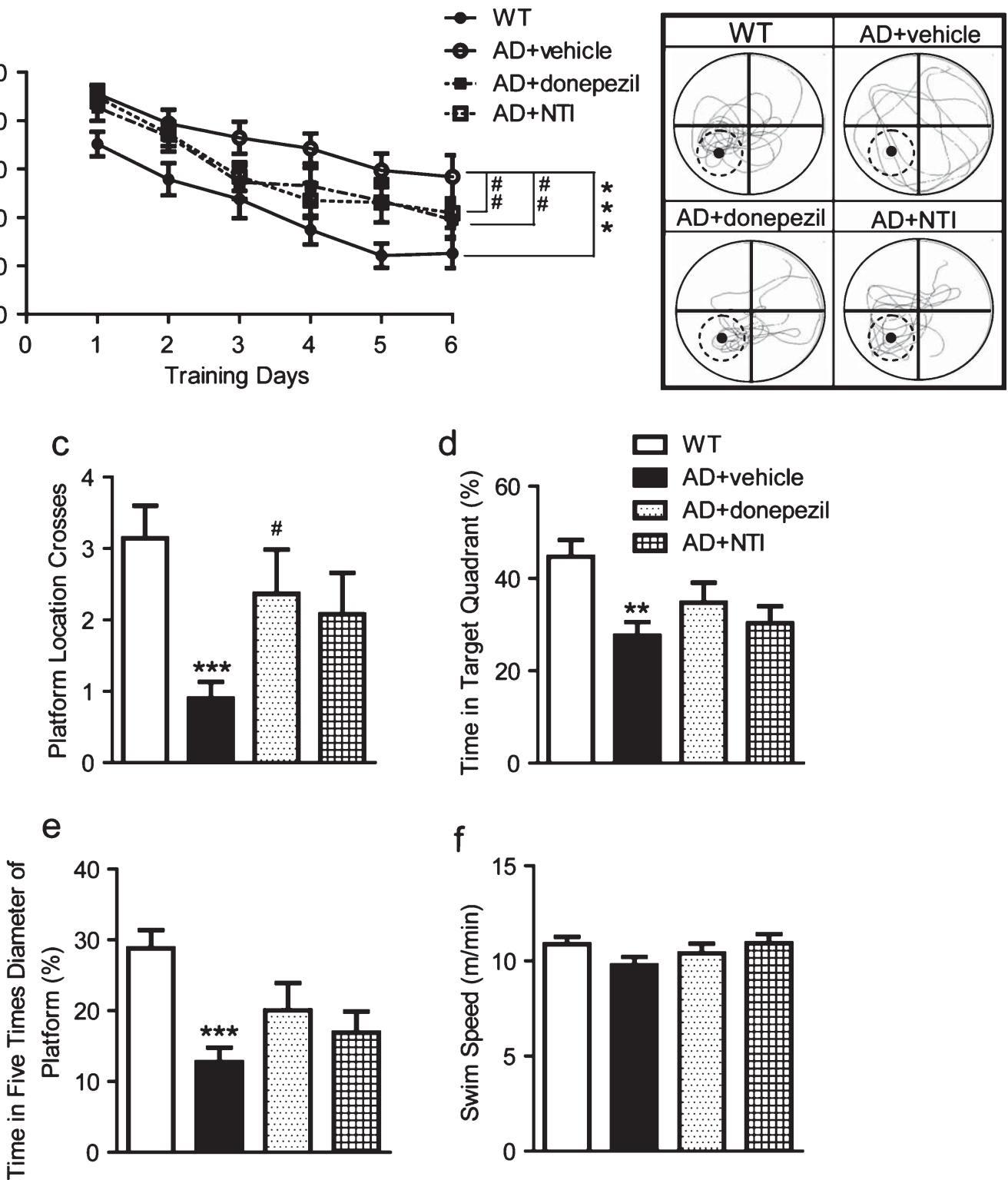

Fig. 3. Donepezil and NTI treatment improve the spatial learning and memory of AßPP/PS1 mice in Morris water maze task. Mice participated in the novel object recognition test were used in this assay. a) The average latency to escape to the hidden platform. $* * * p<0.001$, \#\# $p<0.01$ (two-way ANOVA test). b) Representative swimming paths from different treatment groups. The black dot indicates the previous platform location and the dash line circle indicates the area of five times the platform diameter centered at the platform location. c) The times of platform crossing. d-e) The percent of time mice spent in target quadrant or in the area of five times the platform diameter in the probe trial. $* * p<0.01$ and ${ }^{* * *} p<0.001$ versus WT; ${ }^{*} p<0.05$ versus AD vehicle controls (Student's $t$-test). f) The swimming speed of mice from different treatment groups.

deposition is usually associated with inflammatory responses [22]. In the $\mathrm{AD}$ mouse model, $\mathrm{A} \beta$ plaques are usually surrounded by large numbers of activated astrocytes, which appeared as GFAP positive clusters in brain sections [23]. Compared with vehicletreated AD mice, the NTI-treated group showed a significant reduction in GFAP positive areas (Fig. $4 \mathrm{~b}$ and e), while the donepezil-treated group only displayed a tendency of reduction. These data agree with the previous study that blocking DOR activation leads to decreased $\gamma$-secretase activity and reduced inflammation reaction in brain [21]. However, the lack of a significant reduction of astrocyte activation by donepezil might reflect the character of this 

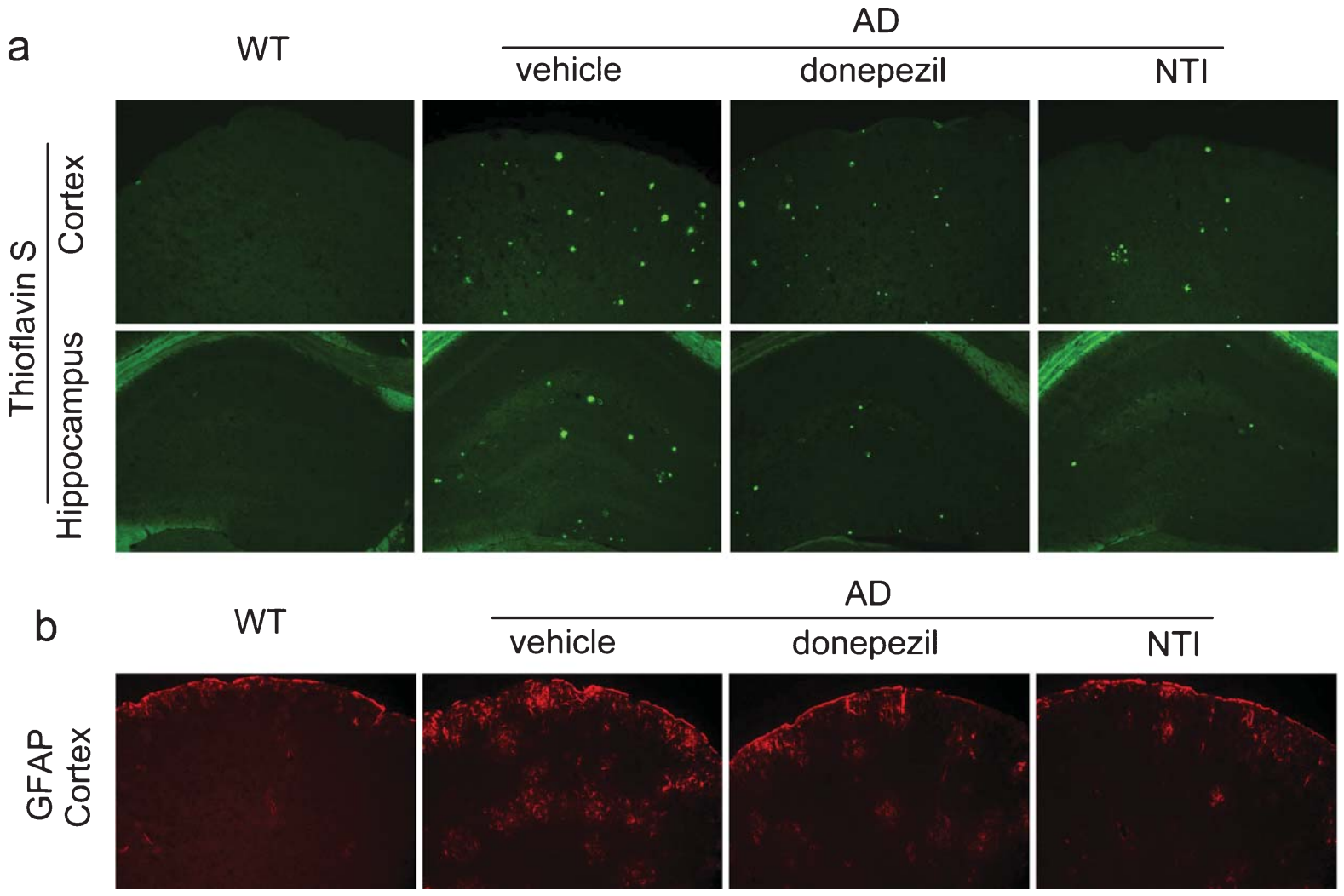

$A D$

C

d
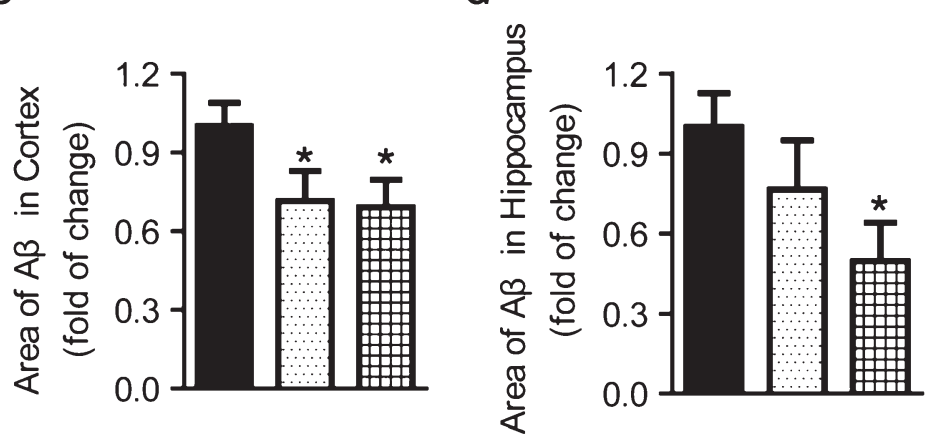

e
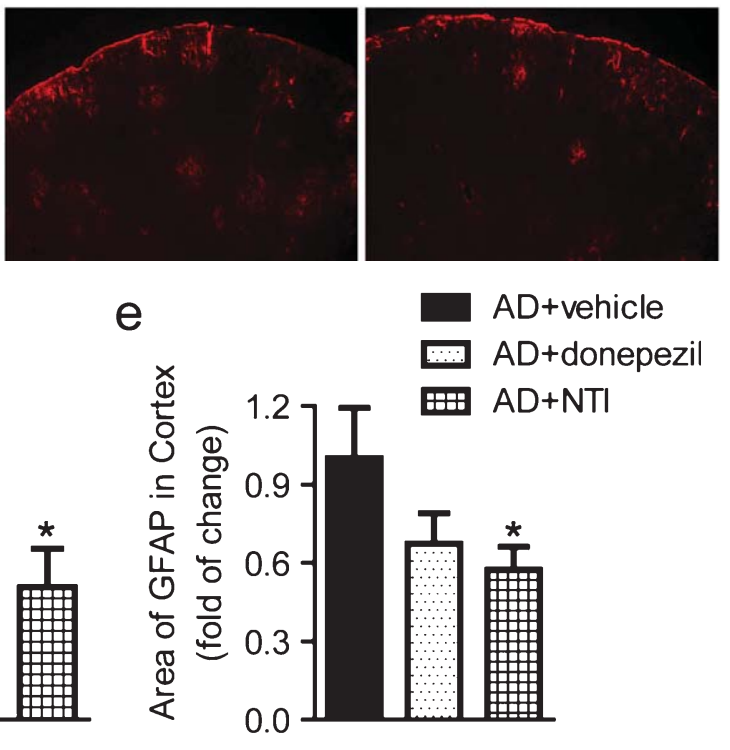

Fig. 4. Pathological analysis of the brain sections from AßPP/PS1 AD mice. a) Thioflavin S staining of amyloid plaques in the cortex and hippocampus. b) Astrocyte activation revealed by GFAP staining in the cortex. c-d) Quantitative analysis of plaque area in a. e) Quantification of GFAP positive area in b. Bars indicate mean \pm S.E.M. $(n=10 \sim 13$ per group). For each mouse, $6 \sim 8$ brain sections from either cortex or hippocampus were analyzed. * $p<0.05$ versus AD vehicle group (Student's $t$-test).

compound as an AchE inhibitor rather than an inflammation inhibitor.

\section{Cognitive deficits of $A D$ mice observed in novel} object recognition correlate well with that found in Morris water maze

A straightforward way to access the correlation between these two assays is to prepare figures with the $\mathrm{X}$-axis and $\mathrm{Y}$-axis representing the performance of novel object recognition and Morris water maze, respectively, and analyzing the data with linear regression. However, as shown in Fig. 5, data from these behavioral tests were rather discrete and were not suitable for regression analysis. Although difficult to draw a regression line from these scattered data point, we were still able to distinguish different distribution patterns of the data generated from AD or WT mice. Most 
a
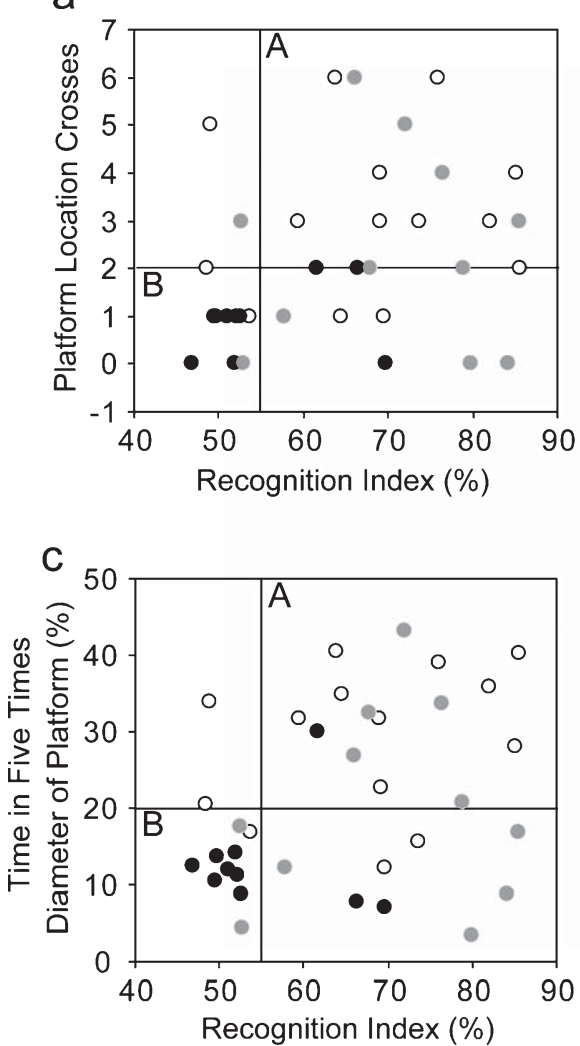

b

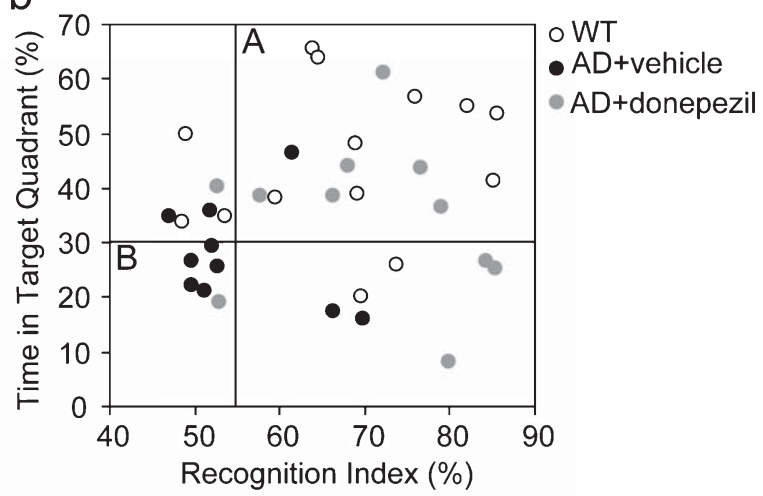

d



Fig. 5. The correlation between the novel object recognition test and Morris water maze test. The novel object recognition indexes of mice from the WT $(n=14)$, AD vehicle group $(n=10)$ and donepezil-treated group $(n=11)$ were plotted against various parameters generated from the Morris water maze test. a) The relationship between the recognition index and number of platform location crosses. b) The relationship between the recognition index and time in target quadrant. c) The relationship between the recognition index and time in the area of five times the platform diameter. d) The relationship between the recognition index and swim speed.

of the data from $\mathrm{AD}$ mice scattered at the lower left field, while most of the data from WT mice located at the upper right field. These distribution patterns remind us of the scatter plots commonly used in FACS data analysis with two fluorescent labeling (double positive located at the upper right field, while double negative located at the lower left field). Therefore, enlightened by the scatter plot from FACS analysis, we analyzed our data in a similar way. According to the distribution pattern of AD and WT mice, each scatter plot can be divided into four quadrants using $55 \%$ (50\% if by chance) of the RI as the separation point on the Xaxis, and 2 times of platform crossing, 30\% (25\% if by chance) of the time in the target quadrant, and $20 \%$ (approximately $10 \%$ if by chance) of the time in the area of five times the platform diameter centered at the platform location as the separation points on the Y-axis. More than $60 \%$ of the WT mice performed well in both novel object recognition and Morris water maze tests and their data fell into quadrant $\mathrm{A}$ in all three types of analysis. Only about $10 \%$ of the WT mice performed poorly in both tests and fell into quadrant B (Fig. 5a-c and Table 1). In contrast, only $10 \%$ of the $\mathrm{AD}$ mice fell into quadrant $A$ and the majority (50-70\%) fell into quadrant B (Fig. 5a-c and Table 1), indicating the poor performance in both assays due to their cognitive defects. When plotting swimming speed against recognition index, no separation patterns of $\mathrm{AD}$ and wild type mice were observed (Fig. 5d).

The drug-treated group was also analyzed in the same plot. As expected, donepezil-treated AD mice showed a distribution pattern more similar to the WT mice rather than the vehicle-treated AD mice. More than $45 \%$ of the donepezil-treated AD mice appeared in quadrant $\mathrm{A}$ and only a small portion (less than $20 \%$ ) remained in quadrant B. Similar result was also obtained with NTI (data not shown), indicating the therapeutic effects of the drug. These results suggest that data generated with the novel object recognition test correlate well with the data from Morris water 
Table 1

The correlation between the novel object recognition test and Morris water maze test. The percentage of animals performed well (corresponding to the quadrant A in Fig. 5a-c) or poorly (corresponding to the quadrant B in Fig. 5a-c) in both tests

\begin{tabular}{|c|c|c|c|c|c|c|c|c|c|}
\hline \multirow{2}{*}{$\begin{array}{l}\text { Correlations } \\
\text { Groups/ } \\
\text { Quadrants }\end{array}$} & \multicolumn{3}{|c|}{$\begin{array}{c}\text { Recognition index \& } \\
\text { Platform location crosses }\end{array}$} & \multicolumn{3}{|c|}{$\begin{array}{l}\text { Recognition index \& } \\
\text { Time in target quadrant }\end{array}$} & \multicolumn{3}{|c|}{$\begin{array}{c}\text { Recognition index \& } \\
\text { Time in five time diameter platform }\end{array}$} \\
\hline & $\begin{array}{l}\text { WT } \\
(\%)\end{array}$ & $\begin{array}{c}\mathrm{AD}+ \\
\text { vehicle }(\%)\end{array}$ & $\begin{array}{c}\mathrm{AD}+ \\
\text { donepezil }(\%)\end{array}$ & $\begin{array}{l}\text { WT } \\
(\%)\end{array}$ & $\begin{array}{c}\mathrm{AD}+ \\
\text { vehicle }(\%)\end{array}$ & $\begin{array}{c}\mathrm{AD}+ \\
\text { donepezil }(\%)\end{array}$ & $\begin{array}{l}\text { WT } \\
(\%)\end{array}$ & $\begin{array}{c}\mathrm{AD}+ \\
\text { vehicle }(\%)\end{array}$ & $\begin{array}{c}\mathrm{AD}+ \\
\text { donepezil }(\%)\end{array}$ \\
\hline $\begin{array}{c}\text { A } \\
\text { Mice performed } \\
\text { well in both test } \\
\text { B }\end{array}$ & 60.7 & 10.0 & 45.5 & 64.3 & 10.0 & 54.5 & 64.3 & 10.0 & 45.5 \\
\hline $\begin{array}{l}\text { Mice performed } \\
\text { poorly in both } \\
\text { test }\end{array}$ & 10.7 & 70.0 & 9.1 & 0 & 50.0 & 9.1 & 7.1 & 70.0 & 18.2 \\
\hline
\end{tabular}

maze, and both assays are suitable to study cognitive deficits in $\mathrm{AD}$ mouse models and to evaluate therapeutic effects of drugs. Since the novel object recognition test is a relatively simple, sensitive, and friendly assay for mice, it could be used as the primary behavioral test for evaluating $\mathrm{AD}$ therapeutic compounds in A $\beta P P / P S 1$ mice.

\section{DISCUSSION}

$\mathrm{AD}$ is a neurodegenerative disease characterized by the depositions of $A \beta$ plaques and the formation of neurofibrillary tangles [24]. In addition to the pathological changes in the brain, deficits in learning and memory are other parameters to assess the progression of AD in animal models. Morris water maze is a classic method to examine the spatial learning and memory in rodents and is widely applied to study the behavioral deficits in AD animal models. However, setting up the water maze in a specific pathogen free animal facility is a complicated procedure and the testing condition is somewhat stressful to the animals. A more simple and friendly behavioral test would be helpful to evaluate a large number of potentially beneficial compounds in $\mathrm{AD}$ animals. In the present study, we carefully compared the novel object recognition test with the Morris water maze, and clearly demonstrated that the novel object recognition test is a facile and sensitive behavioral test in A $\beta P P / P S 1$ AD model.

The novel object recognition test is based on the spontaneous behavior of rodents to explore novelty and is a pure working memory test free of reference [9]. Hippocampus is important in the formation of recognition memory in both human and animals [25]. Perirhinal cortex has also been proposed to play a key role in object recognition [26]. There are two main advantages of the novel object recognition test compared to other behavioral assays. Firstly, it is a relatively simple and friendly test for the animals. It does not require spatial learning and the application of positive or negative reinforcement stimuli. One big problem in water maze or fear conditioning test is the involvement of negative reinforcement stimuli, such as deep water or electric shocks. These stimuli may cause stress or even depression in mice. Stress has been considered as a major risk factor that negatively affects learning and memory [27, 28]. The features of the object recognition test are comparable to the memory test currently used in humans, since human tests are usually conducted in gentle manners. Secondly, the object recognition paradigm requires a shorter time period and is much easier to be repeated. The simple procedure permits a large number of mice to be tested simultaneously. In addition, this test can be introduced repeatedly to the mice to monitor the progression of memory impairment alone with aging or drug therapy. Due to these advantages, the spontaneous novel object recognition test has been used to study learning and memory in a number of disease models. The Tcl mouse model of Down syndrome showed selectively impairment in short-term memory but not long-term memory in the novel object recognition test [29]. The $\mathrm{PAH}^{\text {enu2 }}$ mouse model of phenylketonuria also displayed cognitive deficits in object recognition and early 5-HTP treatment prevented the discrimination deficits [30]. Cognitive deficits in schizophrenia were also detected in novel object recognition test in the STOP Null mouse model [31]. This test has also been used to study the Hurler syndrome [32] and Rett syndrome [33]. In the field of $\mathrm{AD}$, a few reports have shown that novel object recognition is sensitive to test drug effects in $\mathrm{AD}$ animal models $[12,34]$, which is in agreement with our results.

Though simple and straightforward, the experimental conditions of this test should still be carefully optimized. Compared to rats, mice generally have 
much shorter memory retention time (up to 1 hour) [35]. Different mouse stains also displayed variations in the exploration activity and memory retention time [36]. Therefore, it is necessary to test the exploration activity and obtain the memory curve of different $\mathrm{AD}$ mouse models in advance. Other facts, including gender [37] and housing conditions (social isolation or in groups) [38], should also be carefully tested. Another crucial point of the novel object recognition test is the distinction between novelty exploration and object preference. That means the preference of the two pairs of objects used in the experiment should be equally tested in the training session and mice should not explore one pair of the objects more than the other pair. Important features of the objects including shape, texture, height, color, and odor should be evaluated. Previous studies have reported that the rats showed a preference for objects that have affordances for common rat activities [17], so large or tall objects are not recommended to be used in the test. Objects with a complex surface such as having holes on them attract more attentions than objects with a flat surface. And mice tend to spend more time exploring bright objects than the dark ones because they can discriminate different lightness [19]. Interestingly, an object memory test involving the spatial memory, namely object location test, has also been introduced [39]. In the testing phase of this location test, one of the two identical objects in the training phase is moved to a new position.

In summary, the novel object recognition test is a facile behavior test for A $\beta P P / P S 1$ AD mice which relies on animals' innate preference to explore a novel object without the involvement of positive or negative stimuli. The experimental condition is friendly to the animals and is very close to that of human behavioral tests. It could replace the Morris water maze as the primary behavioral test in evaluating new drugs for $\mathrm{AD}$ treatment.

\section{ACKNOWLEDGMENTS}

The authors thank Dr. Chang-sheng Du for his suggestions and discussions. This work was supported by grants from the Ministry of Science and Technology of China (2009ZX09302-001, 2011DFB30010), the National Natural Science Foundation of China (31071227, 81100963, 31171348), and Shanghai Commission of Science and Technology (11DZ2292200).

Authors' disclosures available online (http://www.jalz.com/disclosures/view.php?id=1327).

\section{REFERENCES}

[1] Smith MA (1998) Alzheimer disease. Int Rev Neurobiol 42, $1-54$.

[2] Garcia-Alloza M, Robbins EM, Zhang-Nunes SX, Purcell SM, Betensky RA, Raju S, Prada C, Greenberg SM, Bacskai BJ, Frosch MP (2006) Characterization of amyloid deposition in the APPswe/PS1dE9 mouse model of Alzheimer disease. Neurobiol Dis 24, 516-524.

[3] Holcomb LA, Gordon MN, Jantzen P, Hsiao K, Duff K, Morgan D (1999) Behavioral changes in transgenic mice expressing both amyloid precursor protein and presenilin-1 mutations: Lack of association with amyloid deposits. Behav Genet 29, 177-185.

[4] D'Hooge R, De Deyn PP (2001) Applications of the Morris water maze in the study of learning and memory. Brain Res Brain Res Rev 36, 60-90.

[5] Dineley KT, Xia X, Bui D, Sweatt JD, Zheng H (2002) Accelerated plaque accumulation, associative learning deficits, and up-regulation of alpha 7 nicotinic receptor protein in transgenic mice co-expressing mutant human presenilin 1 and amyloid precursor proteins. J Biol Chem 277, 22768-22780.

[6] Comery TA, Martone RL, Aschmies S, Atchison KP, Diamantidis G, Gong X, Zhou H, Kreft AF, Pangalos MN, Sonnenberg-Reines J, Jacobsen JS, Marquis KL (2005) Acute gamma-secretase inhibition improves contextual fear conditioning in the $\mathrm{Tg} 2576$ mouse model of Alzheimer's disease. J Neurosci 25, 8898-8902.

[7] Ennaceur A, Meliani K (1992) Effects of physostigmine and scopolamine on rats' performances in object-recognition and radial-maze tests. Psychopharmacology (Berl) 109, 321-330.

[8] Chapman PF, White GL, Jones MW, Cooper-Blacketer D, Marshall VJ, Irizarry M, Younkin L, Good MA, Bliss TV, Hyman BT, Younkin SG, Hsiao KK (1999) Impaired synaptic plasticity and learning in aged amyloid precursor protein transgenic mice. Nat Neurosci 2, 271-276.

[9] Ennaceur A, Delacour J (1988) A new one-trial test for neurobiological studies of memory in rats. 1: Behavioral data. Behav Brain Res 31, 47-59.

[10] Dodart JC, Mathis C, Ungerer A (1997) Scopolamine-induced deficits in a two-trial object recognition task in mice. Neuroreport 8, 1173-1178

[11] Alkam T, Nitta A, Mizoguchi H, Itoh A, Nabeshima T (2007) A natural scavenger of peroxynitrites, rosmarinic acid, protects against impairment of memory induced by Abeta(2535). Behav Brain Res 180, 139-145.

[12] Greco SJ, Bryan KJ, Sarkar S, Zhu X, Smith MA, Ashford JW, Johnston JM, Tezapsidis N, Casadesus G (2010) Leptin reduces pathology and improves memory in a transgenic mouse model of Alzheimer's disease. J Alzheimers Dis 19, 1155-1167.

[13] Yuede CM, Zimmerman SD, Dong H, Kling MJ, Bero AW, Holtzman DM, Timson BF, Csernansky JG (2009) Effects of voluntary and forced exercise on plaque deposition, hippocampal volume, and behavior in the Tg2576 mouse model of Alzheimer's disease. Neurobiol Dis 35, 426-432.

[14] Jardanhazi-Kurutz D, Kummer MP, Terwel D, Vogel K, Dyrks T, Thiele A, Heneka MT (2010) Induced LC degeneration in APP/PS1 transgenic mice accelerates early cerebral amyloidosis and cognitive deficits. Neurochem Int 57, 375-382.

[15] Santa-Maria I, Perez M, Hernandez F, Avila J, Moreno FJ (2006) Characteristics of the binding of thioflavin $S$ to tau paired helical filaments. J Alzheimers Dis 9, 279-285.

[16] Zhuang ZP, Kung MP, Wilson A, Lee CW, Plossl K, Hou C, Holtzman DM, Kung HF (2003) Structure-activity 
relationship of imidazo[1,2-a]pyridines as ligands for detecting beta-amyloid plaques in the brain. J Med Chem 46, 237-243.

[17] Chemero A, Heyser C (2005) Object exploration and a problem with reductionism. Synthese 147, 403-423.

[18] Ennaceur A (2010) One-trial object recognition in rats and mice: Methodological and theoretical issues. Behav Brain Res 215, 244-254.

[19] Jacobs GH, Fenwick JA, Williams GA (2001) Cone-based vision of rats for ultraviolet and visible lights. J Exp Biol 204, 2439-2446.

[20] Bevins RA, Besheer J (2006) Object recognition in rats and mice: A one-trial non-matching-to-sample learning task to study 'recognition memory'. Nat Protoc 1, 1306-1311.

[21] Teng L, Zhao J, Wang F, Ma L, Pei G (2010) A GPCR/secretase complex regulates beta- and gammasecretase specificity for Abeta production and contributes to AD pathogenesis. Cell Res 20, 138-153.

[22] Rogers J, Webster S, Lue LF, Brachova L, Civin WH, Emmerling M, Shivers B, Walker D, McGeer P (1996) Inflammation and Alzheimer's disease pathogenesis. Neurobiol Aging 17, 681-686.

[23] Ruan L, Kang Z, Pei G, Le Y (2009) Amyloid deposition and inflammation in APPswe/PS1dE9 mouse model of Alzheimer's disease. Curr Alzheimer Res 6, 531-540.

[24] Selkoe DJ (2001) Alzheimer's disease: Genes, proteins, and therapy. Physiol Rev 81, 741-766.

[25] Barker GR, Warburton EC (2011) When is the hippocampus involved in recognition memory? J Neurosci 31, 1072110731.

[26] Norman G, Eacott MJ (2004) Impaired object recognition with increasing levels of feature ambiguity in rats with perirhinal cortex lesions. Behav Brain Res 148, 79-91.

[27] Park CR, Campbell AM, Diamond DM (2001) Chronic psychosocial stress impairs learning and memory and increases sensitivity to yohimbine in adult rats. Biol Psychiatry 50, 994-1004.

[28] Duncko R, Johnson L, Merikangas K, Grillon C (2009) Working memory performance after acute exposure to the cold pressor stress in healthy volunteers. Neurobiol Learn Mem 91, 377-381.

[29] Morice E, Andreae LC, Cooke SF, Vanes L, Fisher EM, Tybulewicz VL, Bliss TV (2008) Preservation of longterm memory and synaptic plasticity despite short-term impairments in the Tc1 mouse model of Down syndrome. Learn Mem 15, 492-500.

[30] Andolina D, Conversi D, Cabib S, Trabalza A, Ventura R, Puglisi-Allegra S, Pascucci T (2011) 5-Hydroxytryptophan during critical postnatal period improves cognitive performances and promotes dendritic spine maturation in genetic mouse model of phenylketonuria. Int J Neuropsychopharmacol 14, 479-489.

[31] Powell KJ, Hori SE, Leslie R, Andrieux A, Schellinck H, Thorne M, Robertson GS (2007) Cognitive impairments in the STOP null mouse model of schizophrenia. Behav Neurosci 121, 826-835.

[32] Pan D, Sciascia A 2nd, Vorhees CV, Williams MT (2008) Progression of multiple behavioral deficits with various ages of onset in a murine model of Hurler syndrome. Brain Res 1188, 241-253.

[33] Schaevitz LR, Moriuchi JM, Nag N, Mellot TJ, BergerSweeney J (2010) Cognitive and social functions and growth factors in a mouse model of Rett syndrome. Physiol Behav 100, 255-263.

[34] Taglialatela G, Hogan D, Zhang WR, Dineley KT (2009) Intermediate- and long-term recognition memory deficits in $\mathrm{Tg} 2576$ mice are reversed with acute calcineurin inhibition. Behav Brain Res 200, 95-99.

[35] Bertaina-Anglade V, Enjuanes E, Morillon D, Drieu la Rochelle C (2006) The object recognition task in rats and mice: A simple and rapid model in safety pharmacology to detect amnesic properties of a new chemical entity. J Pharmacol Toxicol Methods 54, 99-105.

[36] Sik A, van Nieuwehuyzen P, Prickaerts J, Blokland A (2003) Performance of different mouse strains in an object recognition task. Behav Brain Res 147, 49-54.

[37] Sutcliffe JS, Marshall KM, Neill JC (2007) Influence of gender on working and spatial memory in the novel object recognition task in the rat. Behav Brain Res 177, 117125.

[38] McLean S, Grayson B, Harris M, Protheroe C, Woolley M, Neill J (2010) Isolation rearing impairs novel object recognition and attentional set shifting performance in female rats. J Psychopharmacol 24, 57-63.

[39] Ennaceur A, Michalikova S, Bradford A, Ahmed S (2005) Detailed analysis of the behavior of Lister and Wistar rats in anxiety, object recognition and object location tasks. Behav Brain Res 159, 247-266. 